\title{
Relations Between Task Design and Students' Utilization of GeoGebra
}

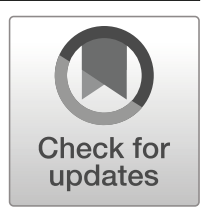

\author{
Jan Olsson ${ }^{1,2}$
}

Published online: 21 March 2019

(C) The Author(s) 2019

\begin{abstract}
This study contributes insights into how task design with different elements of guidance may influence students' utilization of dynamic software for problem solving and reasoning. It compared students' solving of two tasks with different designs supported by the dynamic software GeoGebra. Data analysed examined students' approaches to utilizing GeoGebra, the characteristics of their reasoning and their ability to prove the validity of their solutions after solving the problems. The results showed that students who solved the task with less guidance (without instructions about a specific solving method) were better able to utilize GeoGebra's potential to support their reasoning and problem solving. These students reasoned more creatively and presented more advanced proofs for their solutions than the more guided ones.
\end{abstract}

Keywords GeoGebra · Creative reasoning · Task design · Non-routine tasks

A recurrent question in educational research is whether and how technology may support students' learning mathematics. Dynamic software, in this case GeoGebra, invites students to create and manipulate geometric and algebraic representations features that are considered to have the potential to support problem solving and reasoning (Falcade et al. 2007; Preiner 2008). In situations where students make use of these potentials, they create mathematical objects based on questions or hypotheses. They then interpret, assess and transform the outcomes to proceed in their problem solving. The results, however, are assumed to come from the students' engaging in problem solving and reasoning, and their use of the features of the software to do so. However, it is important to know, rather than simply to assume, what students do when they use dynamic software such as GeoGebra and what guides them in using the

Jan Olsson

jan.olsson@mdh.se

1 Mälardalen University, Box 883, 72123 Västerås, Sweden

2 UMERC, Umeå Research Center for Mathematics Education, Umeå, Sweden 
potential of the software. For example, will the design of a given task influence students' ways of using the software?

Non-routine tasks that students do not have a method for solving, and who are therefore invited to construct (at least parts of) their own method, are beneficial for learning (Brousseau 1997; Jonsson et al. 2014; Kapur and Bielaczyc 2012), as is the requirement that students determine their own way through the solution (Stein et al. 2008). Other studies into the kind of guidance students need to solve non-routine tasks through creating means for solving have included providing hints for getting started, suggesting solving strategies, offering instructions for creating appropriate representations, giving information about important concepts, templates, etc. (Kapur and Bielaczyc 2012). However, some researchers doubt the value of those kinds of support, arguing that, at best, the students will understand the mathematics embedded in the guidance and, at worst, they will just follow the guidance and reach the solution without gaining much (if any) mathematical understanding (Hmelo-Silver et al. 2007; Kapur and Bielaczyc 2012).

Despite research into the potential of dynamic software to support problem solving and reasoning, it is not yet clear how task design may affect students' utilization of the software. In our previous studies (Granberg and Olsson 2015; Granberg 2016; Olsson 2018), we designed tasks to encourage the use of GeoGebra features to support reasoning and problem solving. In these studies, in which the users were GeoGebra novices and the teacher interactions were limited merely to providing instructions for how to use it, we found that guiding students to use general formulas and strategies did not prevent them from engaging in productive reasoning. However, further investigations are needed to clarify whether it is the task design or GeoGebra itself that encourages student engagement in reasoning and problem solving. Therefore, for this study, we designed a task with more specific guidance to compare students' use of GeoGebra between the previous task and the more guided task. The given tasks require the construction of a mathematical rule and the research question is: "Which different methods, if any, do students use to draw on the potential of GeoGebra to support problem solving and reasoning when working with non-routine tasks with different degrees of guidance?"

\section{Background}

The key foci of the research question are GeoGebra's potential to support problem solving and reasoning, and the influence of task design. However, there are at least 20 years of research on different geometric software (e.g. Cabri and Sketchpad) relevant to this study. Therefore, this section begins with a description of earlier research into the potential of dynamic software to support problem solving and reasoning, and concludes with an overview of research into learning about mathematical functions with the use of GeoGebra.

\section{Dynamic Software Supporting Problem Solving and Reasoning}

To construct solving methods and engage in reasoning, students often need support from concrete representations, such as drawings and figures (Preiner 2008). If the 
problem at hand concerns linear functions, students might need to draw and manipulate graphs to explore, for example, the relations between the graphical and algebraic representations of functions. Students might also need tools to investigate the mathematical properties of the visualized representations (Leung 2011). Actively exploring and investigating the properties of linear functions with graphs and illustrations could be easier to do with dynamic software than with pen and paper.

Dynamic software has been shown to support students' problem solving by allowing them to construct graphs simply by entering an algebraic formula (Sedig and Sumner 2006). GeoGebra can also transfer changes from one representation to another. Anything altered in the algebraic representation is automatically adjusted in the graph, and vice versa. Once entered, an object can be precisely adjusted in both representations simultaneously (Preiner 2008). Other system tools such as zoom in and out, construction protocols, angle measurements, and hide and reveal representations support students' problem solving by inviting them to explore and investigate various mathematical properties, processes and relationships (Berger 2011).

Dynamic software such as GeoGebra also creates opportunities for students to consider the mathematics behind the construction of graphs and algebraic expressions (Mariotti 2000), which they may use to justify claims and construct solutions. The ease of creating and adjusting multiple representations supports informal reasoning as an alternative to, or as a way to develop, more formal logical reasoning (Barwise and Etchemendy 1998; Jones 2000). Questions like "How can we increase the slope of graph $\mathrm{x}$ ?" can be explored by constructing, manipulating and comparing several graphs using dynamic software, instead of interpreting just one or two graphs drawn on paper. If these attempts to solve the problem are supported by a prediction of the outcome, they may enhance students' reasoning and justification of their solutions (Hollebrands 2007; Olsson 2018).

\section{Designing Tasks for Problem Solving and Reasoning}

Tasks in mathematics education are generally intended to enhance specific mathematical skills and competencies. However, research frequently notes that textbook tasks often focus on results and answers, which in turn leads to emphasizing procedural skills rather than competencies like problem solving and reasoning (Hiebert and Grouws 2007; Jonassen 2000). Even with tasks explicitly categorized as 'problems' or 'nonroutine', students are often provided with guidelines for constructing appropriate representations of the problem: that is, students do not need to consider which representations are appropriate to support the solution. Instead, they may directly implement the provided instructions to solve the given task (Jonassen 2000). Nonroutine tasks aimed at engaging students in problem solving are also often underpinned with scaffolding, feedback and instructions to secure performance success (Kapur and Bielaczyc 2012). This may affect the way students engage in reasoning.

The use of technology may demand new prerequisites for designing tasks aimed at problem solving and reasoning. The U.S. National Council of Teachers of Mathematics (NCTM 2009) suggests that using technology to link multiple representations of mathematical objects dynamically provides students with opportunities to engage in mathematical sense-making. Such tasks should engage students in problem solving (Santos-Trigo et al. 2016) and invite students to visualize objects and explore dynamic 
variations, by investigating, for example, the areas of triangles with different shapes and a given perimeter.

Leung (2011) argues that for students to benefit from dynamic software, the given tasks should invite them to explore, reconstruct and explain mathematical concepts and relations. He presents a design model for mathematical tasks involving technology. Three modes are introduced: Practice Mode (PM), Critical Discernment Mode (CDM) and Situated Discourse Mode (SDM). These modes mirror the development from learning how to use a tool to gradually realizing the knowledge potential that is embedded in it. Tasks aimed at mathematical exploration may be designed based on these modes in order to encourage interaction between learners and a learning environment including technology.

When approaching a task engaging in PM, the learner needs to establish practices for utilizing the tools for exploration. A scheme for how to use certain tools systematically to achieve a certain purpose will be developed. PM can involve either constructing mathematical objects or manipulating pre-designed objects. In this mode, the learner will develop skills and behaviour for interacting with the tool. The next mode, CDM, builds on PM and arises when learners need to make a critical judgement regarding how to implement the tools in order to observe patterns and invariants. In this mode, the empirical experiences from PM are mathematized: CDM is a precursor to SDM, which serves as a connection to theoretical reasoning and a bridge to formal mathematics.

These three modes may be understood as a nested sequence, in the sense that CDM is a cognitive extension of PM, and SDM a cognitive extension of CDM. To support the different modes when designing tasks, Leung suggests that they should involve conjecturing and providing explanations. Practices will evolve into discernment, while discernment brings about reasoning.

\section{Learning About Functions Using GeoGebra}

To teach the concept of function, lessons must include opportunities to represent, discuss and interpret features of, language involved with and information derived from functions (Carlson 1998). The nature of the tasks, therefore, has a great impact on students' learning about functions. Oehrtman et al. (2008) argue that a strong emphasis on procedural ability is not effective in developing a deep understanding of the notion of function. Instead, the teaching must start in and develop from students' earlier experiences, with the formal definition of functions arising as a conclusion from the learning activity (Vinner and Dreyfus 1989).

GeoGebra's features allow different approaches to investigating linear functions. Not only can it draw a submitted algebraic expression as a graph (Hohenwarter and Jones 2007), one that can be changed by manipulating the expression, it can also derive an algebraic representation from a graph created in the drawing section. The user may then drag and change the graphic representation and observe changes in the algebraic representation (Karadag and McDougall 2011). The $x$-coefficient and the constant term may also be connected to sliders through which the user can easily change the appearance of the graph and explore its relation to the particular algebraic expression. Tasks may be designed to use these features of GeoGebra to encourage students to examine relations between, and properties of, representations of linear functions. 
Gómez-Chacón and Kuzniak (2015) found that students often had difficulties using software tools, which prevented them from constructing the necessary features or links to solve mathematical tasks. In most cases, students needed teacher support to overcome those challenges. If such teacher support does not reveal a solution method, the pedagogical use of software tools can become part of the teaching of mathematical concepts (Leung 2011). Therefore, the design should generate tasks in which software tools are likely to be used. In our previous studies, which used Task 1 in this study (see Fig. 1, below), we found it was possible to instruct students in how to manage GeoGebra without revealing how to solve the task. Short instructions and the availability of the researcher during student activity in relation to the task were sufficient for students to use it to construct solutions to non-routine tasks. The results showed that students engaged in deep reasoning that was supported by GeoGebra.

\section{Framework}

The research question guiding this study asked in which ways students' utilization of GeoGebra was different when solving non-routine tasks with different levels of guidance. It was considered that to support an analysis aiming to answer this research question, a specification of this software's potential to support problem solving and reasoning was required. Furthermore, using it should be interpretable in students' uttered reasoning, and therefore this latter's character is important to explain differences in its utilization. To deepen the analysis, the aspect of proof was considered. Signs of proof were drawn from students' uttered reasoning as were supported by GeoGebra features. Therefore, to examine the students' utilization of GeoGebra, a conceptual framework was constructed by using the following components:

1) a specification of the potential of GeoGebra to support problem solving and reasoning;

2) definitions of imitative and creative reasoning, which have been found to lead to different types of learning and to be enhanced differently by the character of guidance in tasks;

3) categorisation of different types of proof expressed in reasoning, which may be used to explore the variety of proof in the context of the study.

\section{Construct a rule guiding you how to create perpendicular linear functions}

1. Draw a linear function, $\mathbf{y}=\mathbf{m} \mathbf{x}+\mathbf{c}$ in GeoGebra

2. Draw another linear function perpendicular to the first one.

3. Construct a rule that will guide you in choosing the m-values to draw two linear functions that are perpendicular to one another.

4. Test your rule by constructing three pairs of perpendicular linear functions and, if necessary, develop your rule. Make sure you agree with your friend and write the rule down

Fig. 1 Task 1 
In the context of this study, and with respect to the participants' ages, proof is not related to strict logical proofs. Instead, proof is regarded as students' ability to convince themselves and others of the truth in their solutions. Balacheff's (1988) distinction between pragmatic and intellectual proofs will be used to analyse the diversity of students' proofs for their solutions and methods related to different task design.

Points 1 to 3 (above) form the conceptual basis of the analytic method and will be outlined in the following sub-sections. As this study is following up on an earlier quantitative study, in which students who had worked on Task 1 in a post-test significantly outperformed students who had worked on Task 2, both tasks were used again here without change.

\section{The Potential of GeoGebra to Support Problem Solving and Reasoning}

Our previous studies have shown students often need to refer to and compare mathematical representations to engage in reasoning and to be successful problem-solvers (Granberg and Olsson 2015; Olsson 2018). GeoGebra has the potential to provide students with dynamic, algebraic and graphical representations of mathematical objects. It has been found that students use GeoGebra tools to explore and investigate these objects' relations and properties (Preiner 2008; Bu and Schoen 2011; Santos-Trigo et al. 2016). Based on our experiences and literature, in the context of this study (presented below in the "Method" Section), GeoGebra's potential to support problem solving and reasoning is specified in terms of:

- offering a quick and exact dynamically linked transformation and display of representations of mathematical objects that could be utilized to explore relations between the representations;

- offering tools (e.g. to measure, read, organize, and step back and forth through a task-solving session) that could be drawn upon to investigate specific mathematical properties of the visualized representation.

Student use of the potential of GeoGebra was investigated by having them solve tasks using two different designs (see Figs. 1 and 2). Their utilization of it was analysed through interpreting the levels of reasoning observed in their computer actions and conversations. The conceptual basis used for reasoning is outlined in the next subsection.

\section{Imitative and Creative Reasoning}

In mathematics education literature, reasoning is equated with strict proof by some authors, while others define it less narrowly (Balacheff 1988; Ball and Bass 2003). Students in secondary school are introduced to mathematical proofs, but, in everyday classes, they more often perform less strict forms of reasoning. Acceptable reasoning is fostered by the expectations and demands of teachers, textbooks and curricula (Yackel and Hanna 2003). Lithner (2008) describes reasoning as a line of thought adopted to produce assertions and conclusions in order to solve tasks. Inspired by Pólya's (1954) concept of 'plausible reasoning' (distinguishing a more reasonable guess from a less reasonable one), Lithner defined Creative Mathematically-founded Reasoning (CMR) 


\section{Construct a rule gulding you how to create perpendicular linear functions}

A linear function could be presented as $y=m x+c$, i.e. $y=2 x+4$. Use GeoGebra to explore how to choose the $m$-values of two linear functions to make the functions perpendicular to one another.

1) Submit the linear functions, a to $h$, into GeoGebra and write each functions' m-value in column (1) $\mathrm{m}_{1}$-value, in the table.

2) Use GeoGebra to find the linear function that is perpendicular to function a) Write the m-value of that function in column (2) $\mathrm{m}_{2}$-value, in the table. Find the perpendicular function to $b$ ) write its $\mathrm{m}$-value in the column and so on.

3) Multiply $m_{1}$ and $m_{2}$ in each row, write the answer in column (3) $m_{1} \times m_{2}$.

4) Try to create a rule of how to choose two linear functions m-values to make them perpendicular to one another. Make sure you agree with your friend and write the rule down.
a) $y=2 x+2$
b) $y=4 x-2$
c) $y=x-4$
d) $y=-0,25 x-2$
e) $y=-x+3$
f) $y=0,5 x-2$
g) $y=-2 x-3$
h) $y=-0,5 x-6$

\begin{tabular}{|c|c|c|c|}
\hline & $\begin{array}{c}\text { (1) } \\
\mathbf{m}_{1} \text {-value }\end{array}$ & $\begin{array}{c}\text { (2) } \\
\mathbf{m}_{2} \text {-value }\end{array}$ & $\begin{array}{c}\text { (3) } \\
\mathbf{m}_{1} \times \mathbf{m}_{2}\end{array}$ \\
\hline $\mathbf{a}$ & & & \\
\hline b & & & \\
\hline c & & & \\
\hline d & & & \\
\hline c & & & \\
\hline f & & & \\
\hline B & & & \\
\hline h & & & \\
\hline
\end{tabular}

Fig. 2 Task 2

as coming up with original methods for solving mathematical problems, supported by arguments anchored in intrinsic mathematics for prediction and verification. CMR is not necessarily logically strict, but it is constructive through its support for plausible arguments.

Reasoning that is not based on plausible arguments can be imitative, based mainly on given or recalled facts and procedures. Different kinds of imitative reasoning are often suitable for solving routine tasks. Memorized reasoning (MR) relies on recalling an answer (e.g. every step of a proof or facts such as $1 \mathrm{~m}^{3}=1000 \mathrm{dm}^{3}$ ), but more often tasks require procedural calculations (e.g. "draw the graph corresponding to $y=3 x-$ 3 ”). In those cases, algorithmic reasoning (AR) - recalling a set procedure that will solve the task - is usually more suitable. One variant of AR particularly relevant to this study is Guided AR, reasoning based on a given procedure supplied by a person or a text. Reasoning can be seen as a thinking process and/or the product of thinking processes, and it is the latter that we can observe as data (e.g. written solutions, oral 
speech and computer actions), which may be used to examine the type or level of their reasoning while solving problems. The design of this study (which will be presented in detail in the "Method" Section) builds on students solving tasks in pairs with the support of GeoGebra, and their reasoning being visible in their conversations and activity using GeoGebra. Students' reasoning will be characterized through Lithner's framework of imitative and creative reasoning.

\section{Four Types of Proof}

Students may solve tasks equally successfully, but with a variety of types of proof supporting the solution. Balacheff (1988) proposed using qualities of students' proofs for their solutions and methods to explore the variety of proof through solving a task. In this study, his framework of pragmatic and intellectual proofs, as outlined in the next sub-section, was used to characterize students' expressed proofs. He distinguishes students' forms of proof in mathematical practices from strict logical proofs. Student proofs for their solutions are often connected to their own activity generated while solving the problem.

A further difference in student justifications is between pragmatic proofs and intellectual proofs. Pragmatic proofs are directly linked to the students' task-solving actions and are a matter of showing that their method or conjecture works. Intellectual proofs, on the other hand, rest on formulations of, and relations between, properties they are concerned with giving reasons for its truth. Pragmatic proofs can be categorized as either naïve empiricism or the crucial experiment, and intellectual proofs as the generic example or the thought experiment. These four categories are presented in detail below.

Naïve Empiricism This is the very first step in the process of generalization. It makes assertions about truth of a conjecture based on specific examples. For instance, for the question "Is the sum of two odd numbers always even?", a student performs a number of additions (e.g. $1+1,3+3,1+7,5+11$, etc.) and, based on this data alone (each result being specifically even), claims that all sums of two odd numbers are even.

The Crucial Experiment Originally, the expression referred to an experiment designed to prove whether one or another of two hypotheses was valid. Balacheff used the term to represent the assertion of truth based on solving one additional instance of the task, and assuming and asserting that if the conjecture worked for this instance, it would consequently always work for similar instances. For example, a student has the same question as in the example above. Having explored several additions of two odd numbers, with all being even, she states that it is not feasible to try all possible additions. She then decides to try an addition with larger numbers, e.g. $123+235$, and, based on this specific result (358) being even, claims that all sums of two odd numbers are even. The difference between this and naïve empiricism is that here the student explicitly poses the problem of generalization.

The Generic Example It is a characteristic representative of a class of objects which allows for treating a particular instance without losing generality and the student has some awareness of the generality of the specific procedure being used. The difference 
from the crucial experiment is that this type of proof considers the properties of the entire class and their relations. So, if she looks at $5+7$ and says, $5+7=(4+1)+(6+$ $1)=(4+6)+(1+1)=10+2$, which is the sum of two even numbers (and the second one will always be 2) and any other example will work exactly the same way. (This relies on the sum of any two even numbers being even.)

The thought experiment Justification by thought experiment is further detached from a particular representation than the generic example. For instance (same example as above), a student may argue that any odd number is one more than an even number, so adding any two odd numbers is adding two even numbers (which are always even) and then adding 2 to it, which will still keep it even. (This might be seen as a verbal version of $(2 m+1)+(2 n+1)=2 m+2 n+2=2(m+n+1)$.)

\section{Design of the Tasks}

Lithner (2017) proposes that a task engaging students in CMR must: (1) be reasonable for the particular student to construct a solution (creative challenge) and (2) be reasonable with respect to the student's mathematical resources for articulating arguments for the solution method and the solution (justification and conceptual challenge). These principles build on the ideas of Brousseau (1997), that mathematical learning takes place when students have the responsibility to construct (at least parts of) solutions to non-routine tasks for which they do not have a solution method in advance. When including software such as GeoGebra, students may need guidance with respect to earlier experiences of using the software (Gómez-Chacón and Kuzniak 2015).

Task 1 (see Fig. 1) in this study was based on these principles. It was used in our earlier studies (Granberg 2016; Olsson 2018; Olsson and Granberg 2018) and was designed for users with limited experience with GeoGebra. It was shown that they could receive guidance with respect to how to enter algebraic expressions into GeoGebra and in how to use the angle-measuring tool combined with instructions to focus on $m$-values (the $x$-coefficient) and test the solution, but still have to construct parts of the solution themselves.

Task 2 (see Fig. 2) was originally designed for a quantitative study comparing learning outcomes of Tasks 1 and 2 with respect to the characteristics of reasoning (Olsson and Granberg 2018). It was designed based on Lithner's (2017) principle for AR tasks, providing a task-solution method. Compared with Task 1, it offers far more specific guidance, including which functions to enter into GeoGebra, as well as instructions to look for perpendicular pairs of graphs and to focus on the product of gradients $m_{1}$ and $m_{2}$. Task 2 was designed to encourage the Guided AR strategy of following written or oral guidance (Lithner 2008; Palm et al. 2011). In a previous study, tasks designed using the same principles were found to engage students in AR (Jonsson et al. 2014).

In summary, the students' use of GeoGebra and its identified potential to support reasoning and problem solving was used to categorize how students differed in their use of the software and types of their articulated proofs arising from Tasks 1 and 2. The specification of GeoGebra's potential to support problem solving and reasoning in the context for this study is based on our experience from earlier studies (Granberg and 
Olsson 2015; Olsson 2018). Lithner's (2008) framework of creative and imitative reasoning was used to describe students' ways of reasoning, while Balacheff's (1988) types of proof were used to examine the variety of proofs the students produced for their solutions.

\section{Method}

This study is qualitative and was designed as an experiment to compare students' utilization of the specified potential of GeoGebra when solving non-routine tasks with two different task designs with respect to the degree of guidance. The study was undertaken in collaboration with a project group which is investigating learning mathematics in relation to imitative and creative reasoning.

Forty students in school years 7-9 from two different Swedish compulsory schools (one from a major city, one from the countryside) volunteered to participate. Sweden has a compulsory national curriculum and linear functions are introduced in year 9 . The students from year 9 in this study had been taught briefly about aspects of linear functions (although not the conditions under which the graphs of two linear functions will be perpendicular), while those from years 7 and 8 had not. Students from both schools had experiences of technology aids (including GeoGebra), but the major part of mathematics lessons were performed without the aid of technology. Written informed consent was obtained both from each student and from their parents, and all ethical requirements of the Swedish Research Council (SRC 2011) were met.

First, students were randomly divided into two groups, one to solve Task 1 and the other to solve Task 2. Then they were paired randomly, but all were paired within the same year of school. After collecting data from six pairs of students each solving one of the tasks, a decision was taken to collect further data from eight pairs of students solving Task 2 . The reason for that was that the solutions to Task 1 essentially did not deviate from experiences from our earlier studies, while the analysis of the solutions of Task 2 would benefit from an extended sample.

\section{The Two Non-routine Tasks}

The issue of the tasks is to formulate a rule for the conditions under which the graphs of two linear functions will be perpendicular to one another. There are two main sub-tasks to engage in: constructing the necessary examples and identifying the relationship between the corresponding $m$-values that result in perpendicular graphs. For Task 1 , it was considered that students could use the Cartesian table (how to define positions and how to read a graph) in combination with the dual visualization in GeoGebra of algebraic and corresponding graphical representations, in order to construct examples of linear functions with perpendicular graphs. These constructions would serve as references for exploring the circumstances for $m$-values that result in perpendicular graphs.

Due to the students' limited experience with linear functions, the instructions guided them to use the general formula $y=m x+c$. In pilot studies before earlier studies using Task 1 (Granberg 2016; Olsson 2018), it was shown that students with pre-knowledge similar to that of this study's participants did not engage in the task unless they had 
concrete instructions for how to start. Furthermore, Task 1 included instructions guiding students to focus on the $m$-values and to test the rule by constructing three examples. This may have limited the students' utilization of GeoGebra's potential, for example to create and manipulate lines in the graphic field.

On the other hand, creating a graph by submitting an algebraic expression makes the construction less transparent than creating it using the graphing tool. This may promote deeper reasoning for understanding the connection between algebraic and graphic representations (Sedig and Sumner 2006). Tasks involving exploring properties of both $m$ - and $c$-values and concluding which of them affect the slope are highly relevant in this analysis of using GeoGebra. Experiences from earlier studies have shown that students of ages similar to those in this study did engage in such tasks even with these instructions, while pilot studies have shown that without these instructions students often engaged in fruitless trial-and-error strategies. It appeared that they were not able to explore systematically either the $m$ - and $c$-values without guidance. Therefore, the instructions were kept in this study.

Task 2 was originally designed for a quantitative study investigating the learning outcome compared with Task 1 (Olsson and Granberg 2018). The design aimed at removing the incitements for $\mathrm{CMR}$, which is to construct an original solution and formulate arguments for it. More precisely, students solving Task 2 in this study, if they were to follow the instructions, would be provided with the necessary examples and a table useful for identifying the circumstances under which relationships between $m$-values result in perpendicular graphs. Tasks similar to Task 2, with specific instructions and a prepared table, are common in Swedish textbooks. Solving tasks with guiding templates using AR means that students have less need to assess their methods. The arguments students use for their solutions to such tasks are often shallow or not even anchored in mathematics (Lithner 2008). However, if solvers of Task 2 were asked to explain their solution, they would have access to examples similar to those constructed by solvers of Task 1 and might draw on parts of the specified potential of GeoGebra.

\section{Procedure, Data Collection and Method of Analysis}

The study was performed in a separate room from the classroom and involved two students at a time. Students working in pairs had been used in earlier research (see, for example, Schoenfeld 1985; Roschelle and Teasley 1995), which had been found suitable to collect data about problem solving and reasoning. Each pair shared one laptop and the author gave a brief introduction to using GeoGebra (how to enter algebraic functions, how to adjust existing algebraic expressions, how to measure angles, etc.), as well as offering the students all of the technical support necessary for their use of the software while solving the task. The students could ask questions, but were not provided with any further guidelines for solving the task. If students doing Task 1 did not know how to proceed, they were encouraged to explain the way they were thinking; students undertaking Task 2 were encouraged to read the instructions. When students felt they had solved the task, they were asked, "Are you sure the rule works?" and "Can you explain why the rule works?" Their responses were used to examine the quality of their justifications and students could use GeoGebra to support them. 
The students' conversations and screen actions were recorded using the software BB-flashback. The movie files included both the task-solving session itself, as well as the students' answers to the concluding questions. During the task-solving session, the author took notes when students gestured during their conversations. The conversations were transcribed as written text, the computer actions presented within square brackets and gestures were noted within parentheses.

The method of analysis builds on the components presented in the framework (specification of GeoGebra's potential, categorising of reasoning as CMR and AR, and categories of different types of proof). The tasks were designed in relation to principles intended to promote CMR (Task 1) or AR (Task 2). Based on earlier experience, it was expected that reasoning would be observed when students prepared inputs for GeoGebra, assessed outcomes and drew conclusions. Furthermore, students' reasoning in combination with using the software was regarded as a basis for analysis of the pertinence of GeoGebra's features. Therefore, in preparation for analysis, the transcripts were divided into sequences as shown below:

- sub-task $\rightarrow$ formulating input $\rightarrow$ transforming input to output (processed by GeoGebra) $\rightarrow$ using output $\rightarrow$ drawing conclusion.

Both Task 1 and Task 2 required the students have to solve several sub-tasks. In Task 2 , there are explicit suggestions for sub-tasks - for instance, "enter $y=2 x+2$ ". Task 1 does not provide such explicit instructions; instead, the students have to formulate subtasks themselves. In order to monitor whether or not the design of the tasks resulted in the expected student approaches, hypothetical paths for Task 1 and Task 2 were created showing probable ways through the sequences. Figure 3 depicts hypothetical paths through such a sequence for (a) Task 2 and (b) Task 1.

The hypothetical path for a Task 2 sequence is to adapt the sub-task from instructions (1a), enter the suggested input into GeoGebra (2a), then allow GeoGebra to process the input (3), then use the output as it is, merely copying the output as a written or oral answer to the sub-task (4a) and, finally, drawing a conclusion (5).

For Task 1, the hypothetical path starts with formulating a sub-task without guidance ( $1 \mathrm{~b}$; this can be supported by a question, hypothesis or a less-structured exploration, e.g. "What happens if we change the $x$-coefficient?") and constructing and submitting an input complying with the formulated sub-task (2b). GeoGebra processes the input (3), the output is interpreted, assessed or transformed (4b), and a conclusion is drawn (5).

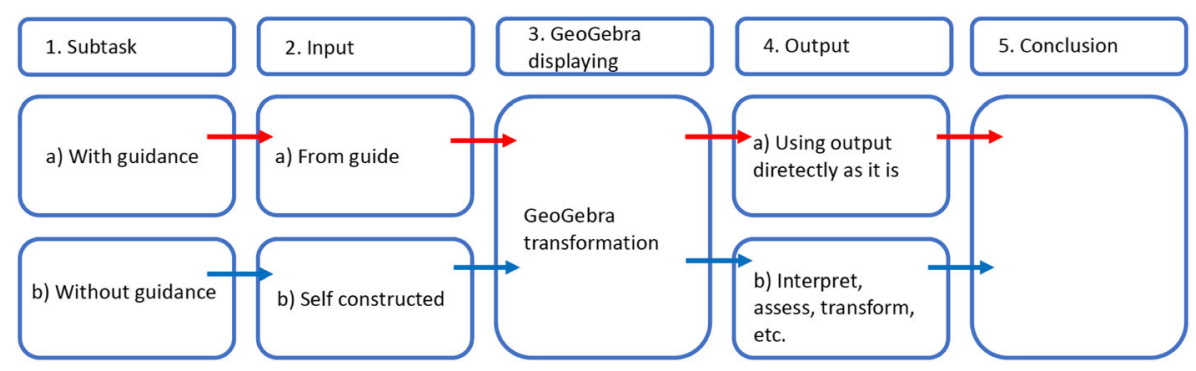

Fig. 3 Hypothetical paths through sequences: red arrows = path for Task 2; blue arrows = path for Task 1 
Since GeoGebra's potential to support reasoning was available to all students, it was of particular interest to examine possible deviations from the hypothetical path of Task 2. The design of Task 2 provides students with opportunities to follow instructions rather than creating solution methods, which is consistent with AR. Furthermore, it provides hints as to what is important, which removes the incitement to assess the outcome. However, students could neglect the proposed input actions and instead construct their own from the main question of the task (path $1 \mathrm{a} \rightarrow 2 \mathrm{~b} \rightarrow 3 \rightarrow 4 \mathrm{~b} \rightarrow 5$ ). They could then follow the path for Task 1. Another possibility is that, after entering the proposed input object, they could interpret, assess or transform the output object (path $1 \mathrm{a} \rightarrow 2 \mathrm{a} \rightarrow 3 \rightarrow$ $4 \mathrm{~b} \rightarrow 5$ ) rather than just transfer it as a solution. Both these approaches entail constructing (parts of) the solution and/or assessing the outcome, which is consistent with CMR. All sequences do not necessarily include all stages. For instance, solvers of both tasks may notice that it is possible to use the existing GeoGebra output (3) to solve a sub-task ( $1 \mathrm{a}$ or $1 \mathrm{~b} \rightarrow 3 \rightarrow 4 \mathrm{a}$ or $4 \mathrm{~b} \rightarrow 5$ ) or they may solve a sub-task without using the software (1a or $1 \mathrm{~b} \rightarrow$ processing without GeoGebra $\rightarrow 5$ ).

The analysis was conducted through the following steps:

1) to structure the data, students' task-solving was categorized as following the hypothetical path for either Task 1 or Task 2 - following the hypothetical path was regarded as reflecting that the intention of either task design was achieved;

2) within these categories, students' task-solving activity was identified and categorized as either using or not using the specified potential of GeoGebra;

3) each student's activity was examined to determine whether the reasoning used was $\mathrm{AR}$ or CMR;

4) the type of the students' proof of their solutions was classified in realtion to Balacheff's categories.

During the progression of the study, the method of analysis was recurrently discussed within the earlier mentioned project group. When the four steps above were set, extracts of transcripts were analysed on trial individually by members of the project group. The group achieved early consensus on steps 1,3 and 4 . We had some initially disagreements about step 2, as some members considered the first specification of GeoGebra's potential to support problem solving and reasoning too generally. Therefore, it was reformulated to connect more closely to the context of the study. The final analysis was conducted by the author.

The first step was to examine whether the students who were given Task 1 followed or deviated from the hypothetical path for Task 1 and whether those solving Task 2 followed or deviated from the hypothetical path for Task 2. Then, to examine students' utilization of GeoGebra's potential, their task-solving activity was divided into sequences (see above). Such sequences typically began with preparing an input for GeoGebra and ended by drawing a conclusion from the software's output (Granberg and Olsson 2015).

After structuring the data into sequences, the next step was to categorize the students' activity and determine how consistent the GeoGebra actions were with the specification of the utilization of GeoGebra's potential. The model in Fig. 3 was used to structure the data, because it was likely that there were substantial differences between the reasoning for and the utilizations of GeoGebra's potential in the task paths for the two tasks. Since the path for Task 1 required more of an inquiry-based approach than 
that for Task 2, it was possible to observe differences in whether and how students chose to use GeoGebra to explore and investigate mathematical properties by transforming and manipulating the mathematical objects.

The reasoning part of the analysis focused on students' speech (suggestions, questions, answers, arguments, etc.) and actions (interaction with GeoGebra and gestures). Lithner's (2008) concepts of reasoning were used to establish whether their reasoning was AR, Guided AR or CMR. When students' utterances and actions were interpreted as merely following the instructions in Task 2, their reasoning was considered Guided AR. When students recalled a memorized procedure, they were categorized as using AR. Finally, students' reasoning was regarded as CMR if they created or re-created a solving strategy (not recalling a whole procedure or, for solvers of guided task, not following the instructions step-by-step), if they presented arguments for why the strategy would work, did work or did not work, and if their arguments were anchored in intrinsic mathematical properties.

The students' proofs for their solutions and methods were investigated at the subtask level while they were solving the task and also when they believed they had formulated a rule, by asking them the question, "Can you explain why the rule works?" All proofs were classified based on one of Balacheff's four types. A proof solely based on the assumption that several examples confirmed their conjecture was categorized as 'naïve empiricism' (Type 1). If the question of generality was raised and their statement was supported by an explicit exploration that verified whether it worked in the identified instance, the proof was categorized as a 'crucial experiment' (Type 2). If the exploration instead investigated the properties and relations of the representations of linear functions and was used to explain why something was true, it was categorized as a 'generic example' (Type 3). The 'thought experiment' (Type 4) was reached when students detached their proof from specific examples, and stated and argued for properties and relations relevant to the conditions under which the graphs of any two linear functions would be perpendicular.

\section{Analysis}

After dividing transcripts into the sequences of sub-task $\rightarrow$ formulating input $\rightarrow$ transforming input to output (processed by GeoGebra) $\rightarrow$ using output $\rightarrow$ drawing conclusion, the sequences were compared with the established hypothetical paths (see Fig. 3). Three categories were identified: solvers of Task 1 following the hypothetical path for Task 1; solvers of Task 2 following the hypothetical path for Task 2; solvers of Task 2 deviating from the hypothetical path for Task 2 . The last category contained three pairs of students. These pairs are interesting as their utilization of GeoGebra, reasoning and justification did not differ from other students solving Task 2 when they were following its hypothetical path. But, after deviating from the path for Task 2, they were more similar to students solving Task 1. The analysis of the Task 2 pairs who deviated from the hypothetical path will be presented in a separate sub-section, focusing on the reasons for, and the consequences of, their deviation. The results of the analysis are summarized in Tables 1 and 2, which provide an overview, before more detailed presentations of each analytical step. 
Table 1 Summary of results of Task 2

\begin{tabular}{llllll}
\hline Students & School year & Rule & Utilization of GeoGebra a & Character of reasoning & Type of proof \\
\hline A1 \& A2 & 9 & $m_{1} \times m_{2}=-1$ & Low & AR & Type 1 \\
B1 \& B2 & 9 & $m_{1} \times m_{2}=-1$ & Low & AR & Type 1 \\
C1 \& C2 & 9 & $m_{1} \times m_{2}=-1$ & Low & AR & Type 1 \\
D1 \& D2 & 9 & -2 & Low & AR & AR \\
E1 \& E2 & 8 & $m_{1} \times m_{2}=-1$ & Low & AR & Type 1 \\
F1 \& F2 & 8 & $m_{1} \times m_{2}=-1$ & Low & AR & AR 1 \\
G1 \& G2 & 8 & $m_{1} \times m_{2}=-1$ & Low & AR & Type 1 \\
H1 \& H2 & 8 & $m_{1} \times m_{2}=-1$ & Low & AR & Type 1 \\
I1 \& I2 & 8 & $m_{1} \times m_{2}=-1$ & Low & Type 1 \\
J1 \& J2 & 7 & $m_{1} \times m_{2}=-1$ & Low & AR & Type 1 \\
K1 \& K2 & 7 & $m_{1} \times m_{2}=-1$ & Low & AR/CMR & Type 1 \\
L1 \& L2 & 9 & $m_{1} \times m_{2}=-1$ & Extended & AR/CMR & Type 2, 3 \\
M1 \& M2 & 8 & $m_{2}=-1 / m_{1}$ & Extended & Type 2, 3 \\
N1 \& N2 & 9 & $m_{1} \times m_{2}=-1$ & Extended & CMR & Type 2, 3 \\
\hline
\end{tabular}

a Using GeoGebra's displays and transformations only as information is denoted as "low". Using these features to investigate properties and relations between representations of linear functions is denotation as "extended"

\section{Categorization of Students' Paths Through Solutions}

Structuring data into sequences and comparing them with the hypothetical path for either task showed that students solving Task 1 essentially followed the hypothetical path for Task 1. However, solvers of Task 1 were also following the hypothetical path for Task 2 at the beginning. The following extract from the transcript of students $\mathrm{O} 1$ and $\mathrm{O} 2$ shows how they implemented the first steps towards solving the unguided task:

1. O1: Okay ... let's start ... just enter something ...

2. $\mathrm{O} 2$ : Well ... $\mathrm{y}$ equals one $\mathrm{x} \ldots$ and plus one will be great ...

Table 2 Summary of results of Task 1

\begin{tabular}{llllll}
\hline Students & $\begin{array}{l}\text { School } \\
\text { year }\end{array}$ & Rule & Utilization of GeoGebra ${ }^{\mathrm{a}}$ & Character of reasoning & Type of proof \\
\hline O1 \& O2 & 7 & $100 / m_{1} /-100=m_{2}$ & Extended & CMR & Type 2 \\
P1 \& P2 & 8 & none & Low & AR & none \\
Q1 \& Q2 & 8 & $m_{2}=-1 / m_{1}$ & Extended & CMR & Type 2, 3 \\
R1 \& R2 & 9 & $m_{2}=-1 / m_{1}$ & Extended & CMR & Type 2, 3 \\
S1 \& S2 & 9 & $m_{2}=-1 / m_{1}$ & Extended & CMR & Type 2, 3 \\
T1 \& T2 & 9 & $m_{1} \times m_{2}=-1$ & Extended & CMR & Type 2, 3 \\
\hline
\end{tabular}

a Using GeoGebra's displays and transformations only as information is denoted as "low". Using these features to investigate properties and relations between representations of linear functions is denoted as "extended" 
3. O1: [enters $y=1 x+1] \ldots$ like that?

4. O2: That's great.

5. O1: Then we must create another linear equation ... then y equals ... maybe $\ldots$

6. O2: This one must go down ... you can enter ...

7. O1: [types $y=x-1$, but does not enter it] ... Can we try this?

8. $\mathrm{O} 2$ : Yes ...

9. O1: [enters it] ... No ...

10. O2: No ... we want it the other way around ... this one goes up one step for every $\mathrm{x} \ldots$ we want it to go down one step for every $\mathrm{x}$.

This extract represents two sequences that contains parts of the hypothetical paths both for Task 1 and for Task 2 . In turns 1-4, the students start by following the instructions for Task 1 and performing the sub-task of deciding what to enter; GeoGebra then transforms the input to a graph and the output is observed and found satisfactory. This sequence essentially follows the hypothetical path for Task $2-$ they follow instructions, but do not interpret the output to draw a conclusion. The choice to submit $y=1 x+1$ is unguided, however. Also, turn 5 in the sequence shown in turns 5-10 is an instruction from Task 1 (draw another linear function perpendicular to the first one). They construct the input themselves. Turns 6-10 then follow the hypothetical path for Task 1, with assessment on turn 9 and a conclusion on turn 10. All five pairs who successfully solved Task 1 initially approached the task by following the first instructions.

Turn 10 shows the students have an idea of how to proceed. The following turns show them continuing to look for the solution:

11. O1: We want the line to decrease when $\mathrm{x}$ increases.

12. O2: Then maybe y equals minus $x$ plus one?

13. O1: Yes ... let's try. [Types in $y=-x+1$ and enters it] ... yes.

14. O2: Yes ... [measures the angle] ... it is ninety degrees.

Turns 10 and 11 connect the sequences. The conclusion in one sequence underpins the formulation of a sub-task for the next sequence. This is typical of the five pairs who successfully solved Task 1 . Often, several sequences were associated with the same sub-task. For example, when students T1 and T2 worked to find linear functions resulting in perpendicular graphs, they agreed to enter $y=-5 x+2$ as a starting point. After investigating the intersection of $y$ at 2 and $x$ at 0.4 , they concluded that the graph decreased five steps on the $y$-axis for every step on the $x$-axis, and that a perpendicular graph should 'do the opposite', increase by five steps on the $x$-axis for every unit step on the $y$-axis. That was elaborated into the idea that the linear function for the graph perpendicular to $y=-5 x+2$ must have an $x$-coefficient $1 / 5$. After stating this was true, they submitted $y=3 x+2$ and $y=-1 / 3 x+2$, and $y=-4 x+2$ and $y=1 / 4 x+2$. In this case, three sequences through the hypothetical path for Task 1 were associated with the same sub-task (find pairs of linear functions with perpendicular graphs).

The work of students $\mathrm{T} 1$ and $\mathrm{T} 2$ and turns 11-14 from $\mathrm{O} 1$ and $\mathrm{O} 2$ follow sequences exemplifying the hypothetical path for Task 1 . After following the first two instructions, all successful solvers of Task 1 created their own sub-tasks, formulated input, assessed, interpreted, and transformed the output, and drew conclusions. Therefore, the pairs who 
successfully solved Task 1 essentially followed the hypothetical path for Task 1 , even though they initially only followed instructions.

Fourteen pairs solved Task 2, eleven of which essentially followed its hypothetical path. The following extract is from students A1 and A2:

1. A1: y equals two $\mathrm{x}$ plus two [reading instructions].

2. A2: [types and enters $y=2 x+2$ ] Like this.

3. A1: Then we write two here [fills in the prepared table].

This sequence follows the hypothetical path for Task 2. The sub-task is adapted from the instructions, they used the suggested input, GeoGebra transforms the algebraic representation into a graph, and the students used the output directly without further interpretation or assessment to write the $x$-coefficient in the prepared table (see Fig. 2). This was repeated until all $x$-coefficients were written into the prepared table. Then A1 and $\mathrm{A} 2$ proceeded as follows:

1. A1: Use GeoGebra to find the linear function that is perpendicular to function a [reading instruction 2 for the guided task].

2. A2: These two [pointing with the cursor at an intersection]. I think we can measure them [measures the angle as $90^{\circ}$. Yes.

3. A1: What do I write?

4. A2: Write beside zero point five [pointing at the table]. The other is minus two. They are perpendicular.

5. A1: All right.

This sequence also follows the hypothetical path for Task 2. Although they discuss the result, they do not assess or interpret the result that the $x$-coefficients of 0.5 and -2 result in perpendicular graphs. The attention is rather on how to fill in the table correctly. After identifying all $x$-coefficients giving perpendicular graphs and writing them pairwise in the prepared table, they immediately started to multiply the $m$-values according to the instructions of Task 2 and formulated the following rule:

1. A1: The rule must be the $\mathrm{x}$ coefficients times each other ...

2. A2: Must be minus one ...

3. A1: Yeah ... that's right.

In this sequence the students did not use GeoGebra. Their conclusion was probably based on noticing that all multiplied examples in the filled-in table were -1 . However, turns 1-3 do not indicate any assessment or interpretation of the result. A1 and A2 essentially follow the hypothetical path for Task 1 and are representative of all eleven pairs who did not deviate from the instructions. That is, they read the instructions, entered the suggested functions into GeoGebra, used the information from GeoGebra to find the perpendicular graphs and fill in the table, followed the instructions to multiply the $x$-coefficients, and drew the conclusion that the rule must be $m_{1} \times m_{2}=-1$.

In the following sub-section, those who solved Task 1 or Task 2 following the hypothetical path are considered separately. 


\section{Utilization of GeoGebra's Potential}

There were significant differences in GeoGebra use between pairs who solved Task 1 or Task 2 and followed the appropriate hypothetical path.

\section{Students Solving the Task 1 Following the Hypothetical Path for Task 1}

Solvers of the Task 1 used GeoGebra's quick and exact transformation and display of representations to investigate specific mathematical interrelations and properties of representations of linear functions. Turns 1-5 shows S1's and S2's efforts to discover the values of the $x$-coefficient that result in a decreasing slope:

1. S2: Maybe y equal to minus two $\mathrm{x}$ will make it ...

2. S1: We can try [writes and enters $y=-2 x-1$ ]. ... No. ... [erases $y=-2 x-1$ ]

3. S2: Maybe x must be less than minus two.

4. S1: We want $y$ to decrease less while $x$ increases more.

5. S2: Maybe y equal to minus $\mathrm{x}$ will work [writes and submits $y=-x-1$ ]. ... No.

6. S1: Let's try minus zero point five [write and submits $y=-0.5 x-1$ ]. ... Yeah.

Turns 2, 5 and 6 show how S1 and S2 utilize GeoGebra's feature to transform the algebraic input to graphic output easily. In the next extract, they compare the $x$ coefficients of two examples:

1. S1: Well, if we go from y equals one $x$ plus one to $y$ equals two $x$ plus three, the $\mathrm{x}$ coefficient increases to doubled, and [if we go] from y equals minus one $\mathrm{x}$ minus one to $\mathrm{y}$ equals minus zero point five $\mathrm{x}$ plus three, the $\mathrm{x}$ coefficient is divided by two.

2. S2: Then we can double the two and divide the minus point five by two.... Let's see. ... $\mathrm{y}$ equals four $\mathrm{x}$ plus three and $\mathrm{y}$ equals minus zero point two five $\mathrm{x}$ plus three [writes and enters].... Yeah.

This shows the students using the displays of algebraic and graphic representations side by side to examine the properties of the $x$-coefficient and the relation between two $x$ coefficients when the graphs are perpendicular. They use the dual display to confirm that the solution on turn 2 results in perpendicular graphs. Characteristic solving patterns in Task 1 included constant use of the angle-measuring tool, hiding and revealing created representations, stepping back and forth through the solution, and adjusting existing representations. For example, T1 and T2 adjusted their existing representations when they thought it would be easier to relate the graphs to the axes if they removed the constant, and could thus place the intersection between graphs at $(0,0)$. This example is discussed further in the subsection headed "Categorization of reasoning".

\section{Students Solving Task 2 Following the Hypothetical Path for Task 2}

The eleven pairs following the hypothetical path for Task 2 typically used GeoGebra's potential only for the quick and exact transformation and display of representations and 
to recognize perpendicular graphs and connect them to the algebraic representations. Some used the angle-measuring tool, while others approximated which intersections were $90^{\circ}$. The following extract is from students $\mathrm{H} 1$ and $\mathrm{H} 2$ :

1. H1: Well ... we start with this [enters and submits $y=2 x+2$ ]. Okay.

2. H2: And then y equals four $\mathrm{x}$ minus two. What is the $\mathrm{m}$ value?

3. H1: It is two.

4. H2: Yes. Two. No, it is four.

5. H1: Four it is.

Turn 1 shows the use of quick and exact transformation. Turns 2-5 show the students focused on the display instead of on the instructions for recognizing the $m$-value. The display on the screen was also used to determine which graphs were perpendicular, as shown in the following extract:

1. H1: I think these two [pointing with the cursor at an intersection]. Wait [uses the measure tool and measures the angle as $90^{\circ}$ ]. Yes.

None of the eleven pairs following the hypothetical path for Task 2 used GeoGebra's potential to explore properties of, and relations between, representations of linear functions.

\section{Categorization of Reasoning}

Students solving the Task 1 essentially engaged in CMR; that is, they constructed the solution method and supported it with arguments. The eleven pairs solving Task 2 following the hypothetical path for Task 2 were considered to engage in Guided AR; that is, they used the instructions as a solution method and their arguments for their solutions were shallow or non-existent.

\section{Students Solving Task 1 Following the Hypothetical Path for Task 1}

Typically, students solving Task 1 discussed what to submit into GeoGebra. The following extract is from students T1 and T2, who created two example pairs: $y=$ $x+2$ with $y=-x+2$ and $y=x-2$ with $y=-x-2$ :

1. T1: So far, we have only tried forty-five degrees, like these [pointing at the screen].

2. T2: To formulate the rule we must have something else. Let's try y equals minus five plus two [enters and submits $y=-5 x+2$ ]. We don't need plus two, do we? [removes +2].

3. T1: No, it does not affect the slope. This one decreases five y for every x. Well, if the other one would be ninety degrees, it should go here [points at $(5,1)]$.

4. T2: That is increasing one $\mathrm{y}$ for five $\mathrm{x}$. Wait, that is one divided by five, zero point two.

Turns 1 and 2 show the students' constructing part of the solution. They realize further references are needed to draw the conclusions necessary to formulate the rule. Turn 2 
includes the statement that the constant is not necessary, which is supported by the argument on turn 3 that it does not affect the slope. Turn 3 includes a claim that the line should intersect at $(5,1)$, which is supported on turn 4 with the argument that $y$ should equal 0.2 , which would increase the line by one $y$ for each five $x$. This is an example of CMR, including constructing a (part of) the solution and supporting it with arguments based on mathematics. The five pairs who successfully solved the unguided tasks showed many examples of engaging in CMR.

\section{Students Solving Task 1 Following the Hypothetical Path for Task 1}

The most frequent type of reasoning in students solving Task 2 was Guided AR; that is, they followed the instructions, submitted them into GeoGebra, and accepted the output as part of the solutions without any supporting arguments (as in the example of students A1 and A2). Students E1 and E2 had the following discussion while searching for perpendicular graphs:

1. E1: Perpendicular ... that is when it is like this [crossing the edges of hands].

2. E2: Yes ... ninety degrees ... these two [pointing at the screen], they are a and e.

3. E1: Then we should write nought point five here [filling in the prepared table].

Turns 1 and 2 include a claim about what is perpendicular, supported by another claim that the angle is $90^{\circ}$. This is not considered CMR; rather, it is shallow and does not support an original solution method. Turn 3 indicates that the purpose of the discussion on turns 1 and 2 is to procced in line with the instructions for the task. This includes guided AR, following instructions, and memorized reasoning, all typical for the ten pairs that reached a correct solution by following the hypothetical path for Task 2 .

\section{Type of Proof}

There were substantial differences in justifications between students solving Task 1 or Task 2. Most (eleven out of fourteen pairs) solvers of Task 2 gave only Type 1 proofs for their solutions, while solvers of the unguided task usually gave Type 2 proofs. Answering the question "Can you explain why the rule works?" solvers of the guided task still only provided Type 1 proofs, while three pairs of the unguided students provided Type 3 proofs and one pair gave Type 3 and partly Type 4 proofs.

\section{Students Solving Task 1 Following the Hypothetical Path for Task 1}

Students who solved the unguided task frequently articulated proof for their steps towards solution at the second Type 1, the crucial experiment. The following extract from students R1 and R2 shows their reasoning about how to calculate $m_{2}$ when $m_{1}$ is known:

1. R1: I mean ... look at these [pointing at $y=5 x$ and $y=-0.2 x$ ]. Point two times five is minus one ... then minus one divided by five is minus zero point two.

2. R2: Yeah. If the first one is four, the other one must be minus one divided by four [types and enters $y=4 x$ and $y=-1 / 4 x$ ]. Yeah, now we know it's working. 
3. R1: The rule must be ... if one line is y equals $\mathrm{mx}$ plus $\mathrm{c}$, the other must be y equals minus one divided by $\mathrm{m}$ plus $\mathrm{c}$.

Turns 1 and 2 indicate they also recognize the relationship between one step on the $y$ axis and the number of steps on the $x$-axis as a fraction. This indication is supported by their submission of the $x$-coefficient as $-1 / 4$ in turn 2 . The claim in turn 1 is justified in turn 2 by the success of a single experiment, which may be an example of a proof on Type 2. But, in this moment, it is not clear whether the students have addressed the problem of generality. When asked "Can you explain why the rule works?", students $\mathrm{R} 1$ and $\mathrm{R} 2$ return to their examples:

1. R1: Well ... we had two examples and saw that if one graph increases more, the other must decrease less.

2. R2: Then we found that one $m$ value could be calculated by dividing minus one by the other.

3. R1: That seems to decrease the other graph correctly ... but we couldn't be sure... so we tried another example.

This indicates that the example could be interpreted on either Type 1 or Type 2. But as they build their conclusion on a correct idea (turn 1) and a reasonable conjecture (turn 3 ), and explicitly express the problem of generalization (turn 3), it is regarded as being Type 2.

Typically, when students were asked "Can you explain why the rule works?", they could not answer directly. Instead, they returned to exploring the examples they had constructed while working with the task, and elaborated their proofs to Type 3, as exemplified by $\mathrm{S} 1$ and $\mathrm{S} 2$ :

1. $\mathrm{S} 1$ : If $\mathrm{x}$ is minus one, then $\mathrm{y}$ is here [pointing to $(-1,10)] \ldots$ and then for $\mathrm{y}=0.1, \mathrm{x}$ is here [pointing to $(10,1)]$.

2. S2: This distance [pointing to $(10,1)$; see Fig. 4 ] and this one [pointing to $(-1,10)$ ] are equal.

3. S1: The difference is that this one goes up one $y$ for every ten $\mathrm{x}$, and this one, the opposite, up ten $\mathrm{y}$ for every minus one $\mathrm{x} \ldots$

4. S2: That is, both have moved one step from each axis [pointing at the distances from the $x$ - and $y$-axes respectively]. It is like the axes kind of turn around and remain perpendicular.

On turns 1 and 2, S1 and S2 recognize the similar distances from the $y$ - and $x$-axes and justify them by referring to how the $m$-values affect the slopes to the axis. On turn 3, S1 explains why the distances are equal, which supports the claim on turn 4 as to why the graphs remain perpendicular, that the equations made them move equal distances from each axis. This may be an example of a Type 3 justification, the generic example. Instead of just stating "it works", they refer to how properties of the $x$ coefficient affect the distances to the $x$ - and $y$-axes. The students do not explicitly articulate that the equal distances they refer to mean that the angles between the graphs and the $y$-axis complement each other to $90^{\circ}$, and it could be questioned whether this is 


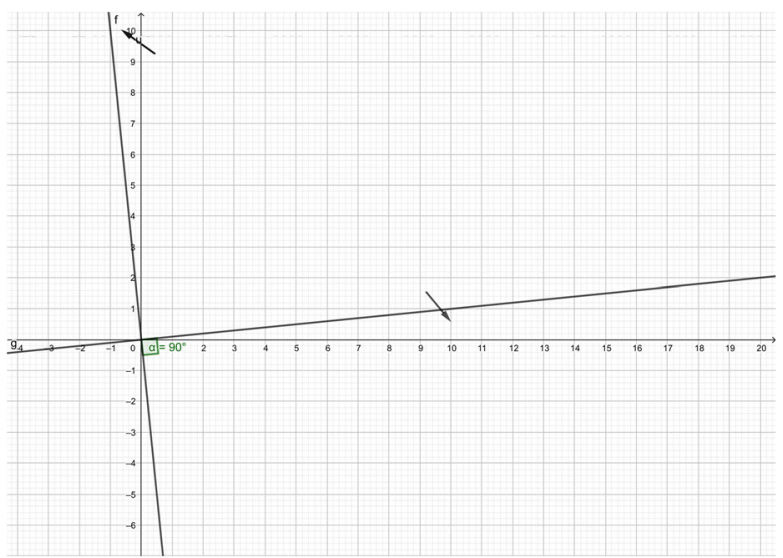

Fig. 4 Arrows point to the distances from the $\mathrm{x}$ - and $\mathrm{y}$-axes, indicating perpendicular lines

an instance of a generic example; whether they actually go beyond simply stating that it works. However, with regard to the age of the students (15 years old) and the fact that they had constructed a couple of examples already before the extract, it is still regarded as an attempt to explain more than merely being content with finding a working method.

Students Q1 and Q2 tried to develop their proof into Type 4, the thought experiment:

1. Q1: Well ... if one $\mathrm{m}$ is four the other must be minus one over four ... and five means minus one over five. Multiplied they [both sets] equal minus one.

2. Q2: Yeah, whatever the $\mathrm{x}$ coefficient is, the other is always minus one divided by the first.

3. Q1: That means they must always be minus one ... that goes for all values.

Turn 1 is still connected to concrete examples. Turn 2 generalizes further, while Turn 3 claims that the conclusion is true for all values. It appears that they have recognized the pattern and realize that with the rule they can create perpendicular graphs for any linear function. But they still do not answer to the why question. They are asked again: why does your rule work?

4. Q1: Well ... one must be positive and one negative ... if we start from $m$ equals one and make $m$ one let's say ten times bigger ... $m$ two must be ten times smaller [it is assumed that Q1 means 1 divided by 10 with respect to the earlier parts of the task solving] ... if one has ten times a slope, the other must have an equally less slope.

5. Q2: Yes ... but one of them must be negative ... and you can take any number.

Q1 returns to using an example, but Q2 claims the generality. With respect to the age of students Q1 and Q2 (both 14 years old), proving that the rule works in general is mathematically advanced, and the students do not explain it very deeply. In Sweden, this level of mathematics is normally taught to 17-18-year- 
olds and, at this age, it is reasonable to demand a more specific proof - for example, considering that the graphs of two linear functions with negative inverted $m$-values form uniform perpendicular triangles parallel to the axis, which may be used to prove that the angle between the graphs must be $90^{\circ}$. For the younger students in this study, however, it was regarded as sufficient that they, with the aid of the visualization on the screen, articulated less specific but nonetheless correct reasons for the generality. However, even though Q1 and Q2 in some sense develop their proof into some generality, it is not fully in Type 4, which for the ages of the students in this study is rare (see Balacheff 1988).

\section{Students Solving Task 2 Following the Hypothetical Path for Task 2}

No student following the hypothetical path for Task 2 articulated any proof while working towards the solution. When asked "Can you explain why the rule works?", no pair solving Task 2 offered any proof other than Type 1, as exemplified by student A1 and A2:

1. A1: Well ... it looks right.

2. Researcher: In what way?

3. A2: They look like they are perpendicular and they are all minus one.

Students $\mathrm{C} 1$ and $\mathrm{C} 2$ had the same question:

1. C1: I don't know.

2. Researcher: Try to explain ...

3. C2: Well ... they are going in opposite directions and they are all minus one.

In both these extracts, the researcher encouraged students to elaborate on their answers, but there were no examples of students developing their proofs beyond Type 1 .

\section{Students Deviating from the Hypothetical Path for Task 2}

Students N1 and N2 deviated from the beginning and followed the hypothetical path for Task 1 through to their solution. Students M1 and M2 followed the instructions, but failed to submit the suggested linear functions correctly and could not formulate a rule. After abandoning the instructions, they solved the task in a way similar to the usual solutions to Task 1. Students L1 and L2 started by following the instructions, but unlike other solvers of Task 2, completed the sub-tasks by investigating the properties of, and relations between, representations of linear functions. This is exemplified in the following extract:

1. L1: Perpendicular to $y$ equals minus $x$ plus three is ... it must be just $\mathrm{x} \ldots$ or $\ldots$

2. L2: I think so ...

3. L1: Then if you do one like this [pointing to the first quadrant ... then you must do another down here [pointing to the second quadrant] ... that would be y equals one $\mathrm{x} . .$. and $\mathrm{y}$ equals minus one $\mathrm{x} . .$. 
The conversation shows that they do not merely accept that the graphs are perpendicular but try to justify why. A bit further in the solution they read the instructions:

1. L1: Multiply $m$ one and $m$ two in each row. What is that?

2. L2: But the formula must be that you invert the $\mathrm{x}$ coefficient and make it negative.

3. L1: That sounds right.

4. L2: Let's try if it works.

Unlike other solvers of Task 2, students L1 and L2 do not just multiply the $x$ coefficients, but instead try to understand what that means for formulating the rule. After formulating a rule (first $m_{2}=-1 / m_{1}$, which was revised into $m_{1} \times m_{2}=-1$ ), they created further examples to support their proof of their rule.

\section{Discussion and Conclusions}

Guided by the research question, "What different methods, if any, do students use to utilize the potential of GeoGebra to support problem solving and reasoning when working with non-routine tasks with different characters of guidance", this study showed important differences between solvers of Task 1 and Task 2 in utilizing the potential of GeoGebra. Beyond the most expected differences (e.g. most students who solved Task 2 followed the instructions and they who solved Task 1 had a more complex path to the solution), it is apparent that students who solved Task 1 to a larger extent also explored properties of and relations between different representation of linear functions.

Looking into detail reveals that Task 1 students more often posed questions and formulated hypotheses. In turn, they engaged with GeoGebra for the purpose of answering questions and verifying/falsifying hypotheses. These actions may also explain differences in the utilization of tools. Task 2 students (who followed instructions) were mainly using the 'angle-measuring tool' and, in some cases, the 'hide-and-reveal feature' to answer the questions given by instructions. Task 1 students used more tools, often for the purpose of examining properties of linear functions - for example the significance of the $x$ coefficient and constant term. When it comes to articulating a proof for the solution, there is a difference between Task 1 and Task 2 solvers in that Task 2 students referred to the results (the table) of following instructions, while Task 1 students often referred to the process towards the solution, creating new actions to support their claims further.

An observation is that it seems like students who solved Task 1 utilized the potential of GeoGebra because they needed to understand properties of linear functions to solve the task. Students who solved Task 2 and utilized the potential of GeoGebra extensively were doing so either if they had made mistakes preventing them from solving the task (students M1 and M2), or if they chose to do so (students L1 and L2, and N1 and N2). That means that Task 2 could be solved without utilizing the potential of GeoGebra and, in this study, most students chose not to. 


\section{Intention and Outcome of Task 2}

Reasonable arguments in favour of the design of Task 2 include preventing students from engaging in fruitless actions that do not bring them any closer to a solution to the task. Guidelines assist them in creating mathematical objects appropriate to support the solution and questions direct them to focus on what is important for creating the solution. Task 2 in this study provided the students with multiple examples of successfully constructed objects and perpendicular graphs, and invited them to explore the relationships between the graphical and algebraic representations of linear functions. Such circumstances have been suggested as beneficial for reasoning (Falcade et al. 2007), problem solving (Santos-Trigo et al. 2016) and developing an understanding of functions (Vinner and Dreyfus 1989).

However, in this study, even though Task 2 students managed to create the rule, they did not utilize GeoGebra's potential to support either creative reasoning (Lithner 2008) or problem solving. On the contrary, these students mainly used GeoGebra to submit the suggested formulas and to measure angles. The students' choice not to utilize the explorative potential of GeoGebra could be explained by their having no reason not to trust that the suggested actions were appropriate for solving the task. When the mathematical objects created by the suggested actions were sufficient to solve the task, students may have seen no reason to investigate them further.

By extension, this means that they never developed systematic procedures with certain purposes, which Leung (2011) characterizes as establishing Practicing Mode (PM). Furthermore, as the following of instructions will generate all the necessary information, there is no incentive to enter Critical Discernment Mode (CDM). Even though they had some issues to consider, they never had to make a critical judgement regarding how to implement the tools to obtain correct information. Finally, formulating the rule for when two linear functions have perpendicular corresponding graphs was unproblematic, which meant there were no actions like making a generalizing conjecture or reasoning to prove or explain. Thus, solvers of Task 2 did not enter Leung's third mode, Situated Discourse Mode (SDM).

Although research often addresses features of dynamic software as supportive of problem solving and reasoning, these results show that, in order to encourage students to utilize the potential of GeoGebra, it is not efficient to provide students with detailed instructions for creating supportive mathematical objects. Task design does seem to be important in encouraging students to utilize the potential of the software. The results indicate that students solving a task with specific guidance are unlikely to use the explorative potential that research has advocated as beneficial, and this may negatively affect students' learning.

\section{Less Specific Guidance Enhances Utilization of GeoGebra}

The results of this study indicate that the design of Task 1 encourages students to utilize the defined potential of GeoGebra. Given general instructions about which mathematical objects to construct, students must consider which specific objects to create and whether they will contribute to the solution. In this study, students repeatedly created graphs by submitting algebraic representations of linear functions into GeoGebra and 
they interpreted the outcomes by investigating properties of, and relations between, algebraic and graphic representations. This illustrates what the literature has suggested as the benefit of using dynamic software in mathematics education (e.g. Hohenwarter and Jones 2007; Preiner 2008) and for learning functions (Carlson 1998; Vinner and Dreyfus 1989). It is also an example of benefitting from GeoGebra's ability to display multiple representations, which is suggested as beneficial for learning functions (Preiner 2008).

In this study, the design of Task 1 (guidance in how to engage in the task, but no instructions for how to create the solution) was shown to engage students in CMR, so that beyond constructing the solution, they created arguments to explain why their solution solved the task. That probably means that they learnt better through understanding their solution better. An important point is that the students who solved Task 1 had (see the "Method" Section) access to instructions showing how to access GeoGebra. This did not seem to affect negatively their utilization of GeoGebra's potential to support problem solving and reasoning. This would indicate that it is important to distinguish between instructions regarding how GeoGebra's tools are used and instructions about how the task can be solved.

The distinction between instructions for using the tools and for solving the task may be important with respect to establishing PM. Without exception, after a few attempts the students who solved Task 1 started creating linear functions with certain purposes. As they created the functions with corresponding graphs on their own, they needed to create and adjust the references to be suitable for observing and understanding patterns supporting the solution. This resonates well with CDM.

With respect to the third of Leung's modes, SDM, solvers of Task 1 certainly had incentives to explain and formulate proofs, but it appears that they largely relied on the visual information provided by GeoGebra. They did not formulate any deeper arguments unless they were encouraged to do so by the author, although there were some attempts to use software tools to create and adjust references for deeper explanations (e.g. students T1 and T2 removed the constant term to have a clearer view of the graphs). However, there is room for development of the instructions for Task 1 here. Encouragement could be included in the written instructions; but, likely more important, it should be the norm always to have students explain solutions. These aspects are issues for further investigation.

\section{The Aspect of Proof}

Neither Task 1 or Task 2 included explicit instructions to the students to formulate a proof for their solutions. However, there were significant differences in the ways students articulated proofs for their solutions, both during the implementation of the task and when they answered the follow-up question. An explanation for these differences could be that students solving Task 1 needed to convince themselves that their solution would work for every sub-task. For example, when calculating the corresponding $x$-coefficient to 3 as $-1 / 3$ in perpendicular graphs, they needed to verify their solution by investigating whether the $x$-coefficients 4 and $-1 / 4$ also will result in perpendicular graphs.

Students who had convinced themselves that the solution worked might be more prepared to explain it on an intellectual level and to consider the mathematics behind its 
construction (Mariotti 2000). That is, they may have chosen to explain why it worked through investigating the properties of and relations between the mathematical representations they had constructed, which might indicate a deeper understanding of the solution. Students who solved Task 2, on the other hand, could merely trust the instructions, follow the guidelines, and reach an answer with no need to assess any of their work.

In this study, when answering why their rule works, solvers of Task 2 articulated proofs for their solutions of Type 1 (naïve empiricism) which may indicate that their understanding of the constructed rule was rather superficial. Furthermore, even though Task 2 required the students to come to a conclusion about the rule for when graphical representations of linear functions are perpendicular, their approach to the task could be interpreted as procedural, which has been found to be ineffective for developing deep understanding (Oehrtman et al. 2008).

This study contributes to the field by showing that it is not sufficient to provide students with dynamic software to promote their engagement in exploring and investigating mathematical objects. It is also important to consider the task design. In school, students are often given tasks with specific guidance because they are thought to be easier with which to engage. However, this study shows how and why guidance included in the design tasks to be solved using dynamic software must not remove the incitements to construct parts of the solution and to formulate arguments and justifications. Thus, it is reasonable to assume that such tasks could lead to a better understanding, which may also indicate better learning outcomes.

Open Access This article is distributed under the terms of the Creative Commons Attribution 4.0 International License (http://creativecommons.org/licenses/by/4.0/), which permits unrestricted use, distribution, and reproduction in any medium, provided you give appropriate credit to the original author(s) and the source, provide a link to the Creative Commons license, and indicate if changes were made.

\section{References}

Balacheff, N. (1988). A study of students' proving processes at the junior high school level. In I. Wirszup \& R. Streit (Eds.), Proceedings of the 15 second UCSMP international conference on mathematics education (pp. 284-297). Reston, VA: National Council of Teachers of Mathematics.

Ball, D., \& Bass, H. (2003). Making mathematics reasonable in school. In J. Kilpatrick, G. Martin, \& D. Schifter (Eds.), A research companion to principles and standards for school mathematics (pp. 27-44). Reston, VA: National Council of Teachers of Mathematics.

Barwise, J., \& Etchemendy, J. (1998). Computers, visualization, and the nature of reasoning. In T. Bynum \& J. Moor (Eds.), The digital phoenix: How computers are changing philosophy (pp. 93-116). London, UK: Blackwell.

Berger, M. (2011). A framework for examining characteristics of computer-based mathematical tasks. African Journal of Research in MST Education, 15(2), 111-123.

Brousseau, G. (1997). Theory of didactical situations in mathematics. Dordrecht, The Netherlands: Kluwer Academic Publishers.

Bu, L., \& Schoen, R. (2011). Model-centered learning: Pathways to mathematical understanding using GeoGebra. Rotterdam, The Netherlands: Sense Publishers.

Carlson, M. (1998). A cross-sectional investigation of the development of the function concept. CBMS Issues in Mathematics Education, 7(2), 114-162.

Falcade, R., Laborde, C., \& Mariotti, M. (2007). Approaching functions: Cabri tools as instruments of semiotic mediation. Educational Studies in Mathematics, 66(3), 317-333. 
Gómez-Chacón, I., \& Kuzniak, A. (2015). Spaces for geometric work: figural, instrumental, and discursive geneses of reasoning in a technological environment. International Journal of Science and Mathematics Education, 13(1), 201-226.

Granberg, C. (2016). Discovering and addressing errors during mathematics problem solving: a productive struggle? The Journal of Mathematical Behavior, 42, 33-48.

Granberg, C., \& Olsson, J. (2015). ICT-supported problem solving and collaborative creative reasoning: exploring linear functions using dynamic mathematics. The Journal of Mathematical Behavior, 37, 4862 .

Hiebert, J., \& Grouws, D. (2007). The effects of classroom mathematics teaching on students' learning. In F. Lester (Ed.), Second handbook of research on mathematics teaching and learning (Vol. 1, pp. 371-404). Greenwich, CT: Information Age.

Hmelo-Silver, C., Duncan, R., \& Chinn, C. (2007). Scaffolding and achievement in problem-based and inquiry learning: a response to Kirschner, Sweller, and Clark (2006). Educational Psychologist, 42(2), 99-107.

Hohenwarter, M., \& Jones, K. (2007). Ways of linking geometry and algebra, the case of GeoGebra. Proceedings of the British Society for Research into Learning Mathematics, 27(3), 126-131.

Hollebrands, K. (2007). The role of dynamic software programs for geometry in the strategies high school mathematics students employ. Journal of Research in Mathematics Education, 34(2), 164-192.

Jonassen, D. (2000). Toward a design theory of problem solving. Educational Technology Research and Development, 48(4), 63-85.

Jones, K. (2000). Providing a foundation for deductive reasoning: students' interpretations when using dynamic geometry software and their evolving mathematical explanations. Educational Studies in Mathematics, 44(1), 55-85.

Jonsson, B., Norqvist, M., Liljekvist, Y., \& Lithner, J. (2014). Learning mathematics through algorithmic and creative reasoning. The Journal of Mathematical Behavior, 36, 20-32.

Kapur, M., \& Bielaczyc, K. (2012). Designing for productive failure. Journal of the Learning Sciences, 21(1), 45-83.

Karadag, Z., \& McDougall, D. (2011). GeoGebra as a cognitive tool: Where cognitive theories and technology meet. In L. Bu \& R. Schoen (Eds.), Model-centered learning: Pathways to mathematical understanding using GeoGebra (pp. 169-181). Rotterdam, The Netherlands: Sense Publishers.

Leung, A. (2011). An epistemic model of task design in dynamic geometry environment. ZDM: The International Journal on Mathematics Education, 43(3), 325-336.

Lithner, J. (2008). A research framework for creative and imitative reasoning. Educational Studies in Mathematics, 67(3), 255-276.

Lithner, J. (2017). Principles for designing mathematical tasks that enhance imitative and creative reasoning. ZDM: Mathematics Education, 49(6), 937-949.

Mariotti, M. (2000). Introduction to proof: the mediation of a dynamic software environment. Educational Studies in Mathematics, 44(1/2), 25-53.

NCTM. (2009). Focus in high school mathematics reasoning and sense making. Reston, VA: National Council of Teachers of Mathematics.

Oehrtman, M., Carlson, M., \& Thompson, P. (2008). Foundational reasoning abilities that promote coherence in students' function understanding. In M. Carlson \& C. Rasmussen (Eds.), Making the connection: Research and teaching in undergraduate mathematics (pp. 27-42). Washington, DC: Mathematical Association of America.

Olsson, J. (2018). The contribution of reasoning to the utilization of feedback from software when solving mathematical problems. International Journal of Science and Mathematics Education, 16(4), 715-735.

Olsson, J., \& Granberg, C. (2018). Dynamic software, task solving with or without guidelines, and learning outcomes. Technology, Knowledge and Learning, 1-18. https://oi.org/10.1007/s10758-018-9352-5. Accessed 5/6/16.

Palm, T., Boesen, J., \& Lithner, J. (2011). Mathematical reasoning requirements in Swedish upper secondary level assessments. Mathematical Thinking and Learning, 13(3), 221-246.

Pólya, G. (1954). Mathematics and plausible reasoning (vols I and II). Princeton, NJ: Princeton University Press.

Preiner, J. (2008). Introducing dynamic mathematics software to mathematics teachers: The case of GeoGebra. Unpublished doctoral dissertation. Salzburg, Austria: University of Salzburg. Retrieved from https://archive.geogebra.org/static/publications/jpreiner-dissertation.pdf. Accessed 5/6/16.

Roschelle, J., \& Teasley, S. (1995). The construction of shared knowledge in collaborative problem solving. In C. O’Malley (Ed.), Computer-supported collaborative learning (pp. 69-197). Berlin, Germany: SpringerVerlag. 
Santos-Trigo, M., Moreno-Armella, L., \& Camacho-Machín, M. (2016). Problem solving and the use of digital technologies within the mathematical working apace framework. ZDM: Mathematics Education, 48(6), 827-842.

Schoenfeld, A. (1985). Mathematical problem solving. Orlando, FL: Academic Press.

Sedig, K., \& Sumner, M. (2006). Characterizing interaction with visual mathematical representations. International Journal of Computers for Mathematical Learning, 11(1), 1-55.

SRC (2011). Ethical principles of research in humanistic and social science. Stockholm, Sweden: Swedish Research Council. http://vr.se. Accessed 10/10/12.

Stein, M., Engle, R., Smith, M., \& Hughes, E. (2008). Orchestrating productive mathematical discussions: five practices for helping teachers move beyond show and tell. Mathematical Thinking and Learning, 10(4), 313-340.

Vinner, S., \& Dreyfus, T. (1989). Images and definitions for the concept of function. Journal for Research in Mathematics Education, 20(4), 356-366.

Yackel, E., \& Hanna, G. (2003). Reasoning and proof. In J. Kilpatrick, G. Martin, \& D. Schifter (Eds.), A research companion to principles and standards for school mathematics (pp. 227-236). Reston, VA: National Council of Teachers of Mathematics.

Publisher's Note Springer Nature remains neutral with regard to jurisdictional claims in published maps and institutional affiliations. 